5. - Free differential calculus. III: Subgroups, Ann. of Math. (2) 64 (1956), $407-419$.

6. - Some problems in knot theory, Topology of 3-manifolds symposium, Prentice-Hall, Englewood Cliffs, N. J., 1962.

7. C. D. Papakyriakopoulos, On Dehn's lemma and the asphericity of knots, Ann. of Math. (2) 66 (1957), 1-26.

8. D. L. Sumners, $O n \pi_{2}$ of $S^{2}$ in $S^{4}$ (to appear).

Florida State University, Tallahassee, Florida 32306

\title{
CLASSIFICATION OF KNOTS IN CODIMENSION TWO
}

\author{
BY RICHARD K. LASHOF AND JULIUS L. SHANESON
}

Communicated by W. Browder, May 31, 1968

Introduction. In this paper we consider smooth knots, i.e., smooth embeddings $\phi: S^{n} \rightarrow S^{n+2}, n \geqq 3$. Two knots $\phi$ and $\eta$ are said to be equivalent if there is a diffeomorphism $f: S^{n+2} \rightarrow S^{n+2}$ such that $f \phi\left(S^{n}\right)=\eta\left(S^{n}\right)$. The embedding $\phi$ extends to an embedding $\phi: S^{n} \times D^{2}$ $\rightarrow S^{n+2}$, and any two such extensions are ambient isotopic relative to $S^{n} \times 0$. Hence if $A=\operatorname{cl}\left(S^{n+2}-\bar{\phi}\left(S^{n} \times D^{2}\right)\right)$, the pair $(A, \partial A)$ is determined up to diffeomorphism by the equivalence class of $\phi$. We call $(A, \partial A)$ the complementary pair, or simply the complement, of the knot $\phi$. In this paper we show that if $\pi_{1} A$, the fundamental group of the knot, is infinite cyclic, then there is at most one knot inequivalent to $\phi$ with complementary pair $(B, \partial B)$ of the same homotopy type as $(A, \partial A)$. This result is of interest because for any $n \geqq 3$ there are many inequivalent knots $\phi: S^{n} \rightarrow S^{n+2}$ with fundamental group $Z$, see for example [12]. (The result also holds in the P.L. case, provided $\phi$ extends to a P.L.-embedding $\bar{\phi}: S^{n} \times D^{2} \rightarrow S^{n+2}$.)

1. Knots with diffeomorphic complements. In [4], Gluck showed that homeomorphisms of $S^{2} \times S^{1}$ are isotopic if and only if they are homotopic and used this result to conclude that there are at most two knots $\phi: S^{2} \rightarrow S^{4}$ with homeomorphic exteriors. In [1], W. Browder studied the pseudo-isotopy classes of diffeomorphisms (and P.L. equivalences) of $S^{1} \times S^{n}$ for $n \geqq 5$. He showed that two P.L. equivalences are pseudo-isotopic if and only if they are homotopic. For the group $D\left(S^{n} \times S^{1}\right)$ of pseudo-isotopy classes of diffeomorphisms, he obtained the exact sequence 


$$
\Upsilon^{n}+\Upsilon^{n+1} \rightarrow D\left(S^{n} \times S^{1}\right) \rightarrow \varepsilon\left(S^{n} \times S^{1}\right) \rightarrow 0,
$$

where $\varepsilon\left(S^{n} \times S^{1}\right)=Z_{2}+Z_{2}+Z_{2}$ is the group of homotopy classes of homotopy equivalences of $S^{1} \times S^{n}$ with itself. Using this result Browder, following Gluck, showed that there are at most two inequivalent knots $\phi: S^{n} \rightarrow S^{n+2}, n \geqq 5$, with diffeomorphic complements. In this section we show that this result is also valid for $n=3$ or 4 .

Proposition 1.1. Let $M^{n+1}, n \geqq 4$, be a P.L. manifold of the same homotopy type as $S^{1} \times S^{n}$. Then $M$ is P.L. homeomorphic to $S^{1} \times S^{n}$.

Proof. For $n \geqq 5$, it follows from the P.L. version of the main theorem of [2] or [3] that $M^{n+1}$ is a P.L. bundle over $S^{1}$ with fiber of the homotopy type of $S^{n}$ and hence P.L. equivalent to $S^{n}$. Therefore $M$ can be obtained from $S^{n} \times I$ by identifying the two boundaries using an orientation preserving P.L. homeomorphism of $S^{n}$ with itself. But such a P.L. homeomorphism is isotopic to the identity.

For $n=4$, this proposition is just the P.L. version of a theorem of [8]. (See also Theorem 2.3 below.)

Corollary 1.2. If $n=4,5$, any smooth $M^{n+1}$ of the same homotopy type as $S^{n} \times S^{1}$ is diffeomorphic to $S^{n} \times S^{1}$.

PRoof. In these dimensions P.L. manifolds have unique smooth structures, see [5].

TheOREM 1.3. For $n=3,4$, any diffeomorphism $d$ of $S^{n} \times S^{1}$ with itself which is homotopic to the identity is pseudo-isotopic to the identity.

Proof. Let $M^{n+2}=D^{n+1} \times S^{1} \cup_{d} D^{n+1} \times S^{1}$. Since $d$ is homotopic to the identity, $M$ has the homotopy type of $S^{n+1} \times S^{1}$, and so is diffeomorphic to $S^{n+1} \times S^{1}$, by Corollary 1.2. Let $g: S^{n+1} \times S^{1} \rightarrow M$ be a diffeomorphism. Writing $S^{n+1} \times S^{1}=D^{n+1} \times S^{1} \cup D^{n+1} \times S^{1}$, we may assume (by the tubular neighborhood theorem and a Whitney embedding theorem) that $g$ carries the first summand in the decomposition of $S^{n+1} \times S^{1}$ into the first summand in the decomposition of $M$ and that its restriction to these summands is a $\mathrm{SO}(n+1)$-bundle map. Hence, after restricting to the second summands and composing with an $\mathrm{SO}(n+1)$-bundle map, we get a diffeomorphism $h: D^{n+1} \times S^{1}$ $\rightarrow D^{n+1} \times S^{1}$ extending $d$; i.e., $h(x, y)=d(x, y)$ for $x$ in $\partial D^{n+1}$ and $y$ in $S^{1}$. Let $D_{0}=\frac{1}{2} D^{n+1}$ be the disk of radius $\frac{1}{2}$. Then by the tubular neighborhood theorem again, we can also insist that $h\left(D_{0} \times S^{1}\right)=D_{0} \times S^{1}$ and that $h \mid D_{0} \times S^{1}$ is an $\mathrm{SO}(n+1)$-bundle map. Hence $d$ is pseudoisotopic to a bundle map $\partial D_{0} \times S^{1} \rightarrow \partial D_{0} \times S^{1}$, which represents $\beta \in \pi_{1}(\operatorname{SO}(n+1))=Z_{2}$, say. Since $d$ is homotopic to the identity, $\beta=0$, 
(since the nontrivial element of $\pi_{1}(\mathrm{SO}(n+1))$ represents a nontrivial element of $\varepsilon\left(S^{n} \times S^{1}\right)$, by [1], for example). Hence $d$ is pseudo-isotopic to the identity.

CoRollary 1.4. For $n=3,4$, let $D\left(S^{n} \times S^{1}\right)$ be the group of pseudoisotopy classes of diffeomorphisms of $S^{n} \times S^{1}$ into itself. Then the natural map $\mathrm{D}\left(S^{n} \times S^{1}\right) \rightarrow \mathcal{E}\left(S^{n} \times S^{1}\right) \cong Z_{2}+Z_{2}+Z_{2}$ is an isomorphism.

Proof. By Theorem 1.3, it is monic, and each generator of $\varepsilon\left(S^{n} \times S^{1}\right)$ is represented by a diffeomorphism. (See [1].)

REMARK. For all $n \geqq 3$, arguments similar to the above can also be used to show that any homotopic P.L. homeomorphisms of $S^{n} \times S^{1}$ to itself are (P.L.) pseudo-isotopic.

The arguments of [1] can now be extended to lower dimensions by using Corollary 1.4. This yields the main result of this section.

THEOREM 1.5. Let $n \geqq 3$. Then there are at most two inequivalent $k$ nots $\phi: S^{n} \rightarrow S^{n+2}$ with diffeomorphic complements.

\section{Knots with complements of the same homotopy type.}

THEOREM 2.1. Let $n \geqq 3$. Let $\phi_{i}: S^{n} \times D^{2} \rightarrow S^{n+2}, i=1,2$, be smooth (or P.L.) embeddings. Let $A_{i}=\operatorname{cl}\left(S^{n+2}-\operatorname{Im} \phi_{i}\right)$ and suppose that $\pi_{1} A_{i}=Z$. Then if $\left(A_{1}, \partial A_{1}\right)$ and $\left(A_{2}, \partial A_{2}\right)$ have the same homotopy type (as pairs), $A_{1}$ and $A_{2}$ are diffeomorphic (resp. P.L. equivalent).

CoRollary 2.2. If $\phi: S^{n} \rightarrow S^{n+2}, n \geqq 3$, is a smooth knot with fundamental group $\boldsymbol{Z}$ and complement $(A, \partial A)$, then there is at most one inequivalent knot with exterior $(B, \partial B)$ of the same homotopy type as $(A, \partial A)$,

Corollary 2.2 follows immediately from Theorem 2.1. We recall that in case $A$ has the homotopy type of a circle, $\phi$ is actually unknotted. (See [6] and [9].)

Proof of Theorem 2.1. We concentrate on the smooth case. The P.L. case can be handled by similar methods. First assume $n \geqq 4$. By Alexander duality and the universal coefficient theorem, $H^{i}(A ; G)=0$ for $j \geqq 2$ and $H^{j}(A ; G)=G$ for $j=0,1, G$ any abelian group. Now let $h:\left(A_{1}, \partial A_{1}\right) \rightarrow\left(A_{2}, \partial A_{2}\right)$ be a homotopy equivalence of pairs, and let $\eta_{h} \in\left[A_{2} ; F / O\right]$ be the "characteristic $F / O$-bundle" of $h$. (See [7] or [11].) $F / O$ is connected and $\pi_{1}(F / O)=0$, and so it follows from Theorem 3 of Chapter 8,84 of $[10]$, that $\left[A_{2} ; F / O\right]=H^{2}\left(A_{2} ; \pi_{2}(F / O)\right)$ $=H^{2}\left(A_{2} ; Z_{2}\right)=0$. So $\eta_{h}=0$. ( $\left[A_{2} ; F / O\right]=$ homotopy classes of maps of $A_{2}$ into $F / O$.) This means that there is a tangential cobordism of $\left(A_{1}, \partial A_{1}\right)$ with $\left(A_{2} ; \partial A_{2}\right)$; i.e. there is a parallelizable $W^{n+1}$ with 
$\partial W=A_{1} \cup \partial_{0} W \cup A_{2}, \partial_{0} W$ a cobordism of $\partial A_{1}$ with $\partial A_{2}$, and a retraction $r:(W, \partial W) \rightarrow\left(A_{2}, \partial A_{2}\right)$ such that $r \mid\left(A_{1}, \partial A_{1}\right)$ is homotopic to $h$. (See [7], [8] or [13].) Now, according to Wall [14] (see also 7.4 and 7.5 of [13]), one can perform surgery relative to $A_{1} \cup A_{2}$ (i.e. without doing any modifications on $A_{1} \cup A_{2}$ ) to get an $s$-cobordism. (This uses the fact that $\pi_{1} A_{2}=Z$.) Thus we get an $s$-cobordism of $\left(A_{1}, \partial A_{1}\right)$ with $\left(A_{2}, \partial A_{2}\right)$, and so the relative $s$-cobordism theorem applies to prove 2.1 for $n \geqq 4$.

Now take $n=3$. Then we have to use the following result from [8].

THEOREM 2.3. Let $M$ be obtained from $S^{5}$ by surgery on an embedded $S^{3}$. Then any manifold of the same homotopy type as $M$ is diffeomorphic to $M$.

Assuming Theorem 2.3, let $h:\left(A_{1}, \partial A_{1}\right) \rightarrow\left(A_{2}, \partial A_{2}\right)$ be a homotopy equivalence. Since every homotopy equivalence of $S^{1} \times S^{3}$ with itself extends to a homotopy equivalence of $S^{1} \times D^{4}$ with itself, it is easy to see that there is a homotopy equivalence $k: A_{1} \cup_{1}^{\phi 0} D^{4} \times S^{1}$ $\rightarrow A_{2} \cup_{\phi_{2}} D^{4} \times S$. Hence by Theorem 2.3 , these manifolds are diffeomorphic. Using a Whitney theorem and the tubular neighborhood theorem again, it follows that there is a diffeomorphism of these manifolds which restricts to a diffeomorphism of $A_{1}$ with $A_{2}$. (Note that the $S^{1}$ 's in the second summands represent generators of the respective fundamental groups of these manifolds.)

REMARK. The above proof of 2.1 for $n \geqq 4$ is essentially a part of the proof that if $(M, \partial M)$ is a smooth manifold pair such that the inclusion induces an isomorphism of $\pi_{1}(\partial M)$ with $\pi_{1} M$, then the "concordance classes of homotopy smoothings of $(M, \partial M)$ " are in 1-1 correspondence with $[M ; F / O]$ via the map induced by taking the "characteristic $F / O$-bundle" of a homotopy equivalence. This result is discussed in the simply-connected case in [7] and [11].

Note. Theorem 2.1 , for $n \geqq 5$, was proved for fibred knots by W. Browder (Manifolds with $\pi_{1}=Z$, Bull. Amer. Math. Soc. 72 (1966), 238-244, Corollary 2.4). Browder informs us that the requirement that the knots be fibred can be eliminated in his approach using recent results of Farrell-Hsiang.

\section{REFERENCES}

1. W. Browder, Diffeomorphisms of 1-connected manifolds, Trans. Amer. Math. Soc. 128 (1967), 155-163.

2. W. Browder and J. Levine, Fibering a manifold over a circle, Comment. Math. Helv. 40 (1965), 153-160.

3. F. T. Farrell, The obstruction to fibering a manifold over a circle, Mimeographed Notes, Yale University, New Haven, Conn., 1968. 
4. H. Gluck, The embedding of two-spheres in the four-sphere, Bull. Amer. Math. Soc. 67 (1961), 586-589.

5. R. Lashof and M. Rothenberg, Microbundles and smoothing, Topology 3 (1965), 357-388.

6. J. Levine, Unknotting spheres in codimension two, Topology 4 (1966), 9-16.

7. C. R. Rourke, The Hauptvermutung according to Sullivan, Mimeographed Notes, Institute for Advanced Study, Princeton, N. J., 1967.

8. J. L. Shaneson, Wall's surgery obstruction groups and classification of 5-manifolds, Thesis, University of Chicago, Chicago, Ill., 1968.

9. - Embeddings of spheres in spheres of codimension two and h-cobordisms of $S^{1} \times S^{3}$, Bull. Amer. Math. Soc. 75 (1968), 972-973.

10. E. H. Spanier, Algebraic topology, McGraw-Hill, New York, 1966.

11. D. Sullivan, Triangulating homotopy equivalences, Thesis, Princeton University, Princeton, N. J., 1966.

12. D. L. Sumners, Higher-dimensional slice knots, Thesis, Pembroke College, Providence, R. I., 1967.

13. C. T. C. Wall, Surgery of nonsimply connected manifolds, Ann. of Math. (2) 84 (1966), 217-276.

14. - Surgery of nonsimply connected manifolds, Mimeographed Notes, University of Liverpool, Liverpool, England, 1967.

University of Chicago, Chicago, Illinois 60608 